Article

\title{
Neutrosophic Triplets in Neutrosophic Rings
}

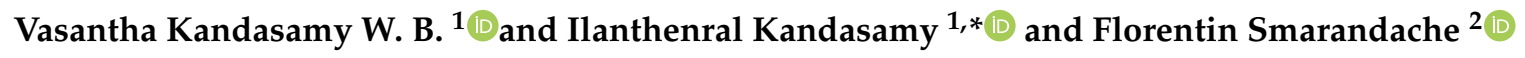 \\ 1 School of Computer Science and Engineering, VIT, Vellore 632014, India; vasantha.wb@vit.ac.in \\ 2 Department of Mathematics, University of New Mexico, 705 Gurley Avenue, Gallup, NM 87301, USA; \\ smarand@unm.edu \\ * Correspondence: ilanthenral.k@vit.ac.in
}

Received: 25 May 2019; Accepted: 18 June 2019; Published: 20 June 2019

\begin{abstract}
The neutrosophic triplets in neutrosophic rings $\langle Q \cup I\rangle$ and $\langle R \cup I\rangle$ are investigated in this paper. However, non-trivial neutrosophic triplets are not found in $\langle Z \cup I\rangle$. In the neutrosophic ring of integers $Z \backslash\{0,1\}$, no element has inverse in $Z$. It is proved that these rings can contain only three types of neutrosophic triplets, these collections are distinct, and these collections form a torsion free abelian group as triplets under component wise product. However, these collections are not even closed under component wise addition.
\end{abstract}

Keywords: neutrosophic ring; neutrosophic triplets; idemponents; special neutrosophic triplets

\section{Introduction}

Handling of indeterminacy present in real world data is introduced in [1,2] as neutrosophy. Neutralities and indeterminacies represented by Neutrosophic logic has been used in analysis of real world and engineering problems [3-5].

Neutrosophic algebraic structures such as neutrosophic rings, groups and semigroups are presented and analyzed and their application to fuzzy and neutrosophic models are developed in [6]. Subsequently, researchers have been studying in this direction by defining neutrosophic rings of Types I and II and generalization of neutrosophic rings and fields [7-12]. Neutrosophic rings [9] and other neutrosophic algebraic structures are elaborately studied in [6-8,10,13-17]. Related theories of neutrosophic triplet, duplet, and duplet set were developed by Smarandache [18]. Neutrosophic duplets and triplets have fascinated several researchers who have developed concepts such as neutrosophic triplet normed space, fields, rings and their applications; triplets cosets; quotient groups and their application to mathematical modeling; triplet groups; singleton neutrosophic triplet group and generalization; and so on [19-36]. Computational and combinatorial aspects of algebraic structures are analyzed in [37].

Neutrosophic duplet semigroup [23], classical group of neutrosophic triplet groups [27], the neutrosophic triplet group [12], and neutrosophic duplets of $\left\{Z_{p n}, \times\right\}$ and $\left\{Z_{p q}, \times\right\}$ have been analyzed [28]. Thus, Neutrosophic triplets in case of the modulo integers $Z_{n}(2<n<\infty)$ have been extensively researched [27].

Neutrosophic duplets in neutrosophic rings are characterized in [29]. However, neutrosophic triplets in the case of neutrosophic rings have not yet been researched. In this paper, we for the first time completely characterize neutrosophic triplets in neutrosophic rings. In fact, we prove this collection of neutrosophic triplets using neutrosophic rings are not even closed under addition. We also prove that they form a torsion free abelian group under component wise multiplication.

\section{Basic Concepts}

In this section, we recall some of the basic concepts and properties associated with both neutrosophic rings and neutrosophic triplets in neutrosophic rings. We first give the following 
notations: $I$ denotes the indeterminate and it is such that $I \times I=I=I^{2} . I$ is called as the neutrosophic value. $Z, Q$ and $R$ denote the ring of integers, field of rationals and field of reals, respectively. $\langle Z \cup I\rangle=\left\{a+b I \mid a, b \in Z, I^{2}=I\right\}$ is the neutrosophic ring of integers, $\langle Q \cup I\rangle=\{a+b I \mid a, b \in Q$, $\left.I^{2}=I\right\}$ is the neutrosophic ring of rationals and $\langle R \cup I\rangle=\left\{a+b I \mid a, b \in R, I^{2}=I\right\}$ is the neutrosophic ring of reals with usual addition and multiplication in all the three rings.

\section{Neutrosophic Triplets in $\langle Q \cup I\rangle$ and $\langle R \cup I\rangle$}

In this section, we prove that the neutrosophic rings $\langle Q \cup I\rangle$ and $\langle R \cup I\rangle$ have infinite collection of neutrosophic triplets of three types. Both collections enjoy strong algebraic structures. We explore the algebraic structures enjoyed by these collections of neutrosophic triplets. Further, the neutrosophic ring of integers $\langle Z \cup I\rangle$ has no nontrivial neutrosophic triplets. An example of neutrosophic triplets in $\langle Q \cup I\rangle$ is provided before proving the related results.

Example 1. Let $S=\langle Q \cup I\rangle,+, \times($ or $\langle R \cup I\rangle,+, \times)$ be the neutrosophic ring. If $x=a-a I \in$ $S(a \neq 0)$, then

$$
y=\frac{1}{a}-\frac{I}{a} \in S
$$

is such that

$$
x \times y=(a-a I) \times\left(\frac{1}{a}-\frac{I}{a}\right)=1-I-I+I=1-I .
$$

Thus, for every $x=a-a I$, of this form in $S$ we have a unique $y$ of the form

$$
\frac{1}{a}-\frac{I}{a}
$$

such that $x \times y=1-I$. Further, $1-I \in S$ is such that $1-I \times 1-I=1-I+I-I=1-I \in S$. Thus, these triplets

$$
\left\{a-a I, 1-I, \frac{1}{a}-\frac{I}{a}\right\} \text { and }\left\{\frac{1}{a}-\frac{I}{a}, 1-I, a-a I\right\}
$$

form neutrosophic triplets with $1-I$ as a neutral element.

Similarly, for aI $\in S(a \neq 0)$, we have a unique

$$
\frac{I}{a} \in S \text { such that } a I \times \frac{I}{a}=I
$$

and $I \times I=I$ is an idempotent. Thus,

$$
\left\{a I, I, \frac{I}{a}\right\} \text { and }\left\{\frac{I}{a}, I, a I\right\}
$$

are neutrosophic triplets with I as the neutral element.

First, we prove $\langle Q \cup I\rangle$ and $\langle R \cup I\rangle$ have only $I$ and $1-I$ as nontrivial idempotents as invariably one idempotents serve as neutrals of neutrosophic triplets.

Theorem 1. Let $S=\langle Q \cup I\rangle,+, \times($ or $\{\langle R \cup I\rangle,+, \times\})$ be a neutrosophic ring. The only non-trivial idempotents in $S$ are I and $1-I$.

Proof. We call 0 and $1 \in S$ as trivial idempotents. Suppose $x \in S$ is a non-trivial idempotent, then $x=a I$ or $x=a+b I \in S(a \neq 0, b \neq 0)$. Now, $x \times x=a I \times a I=a^{2} I\left(\right.$ as $\left.I^{2}=I\right)$; if $x$ is to be an idempotent, we must have $a I=a^{2} I$; that is, $\left(a-a^{2}\right) I=0(I \neq 0)$, thus $a^{2}=a$. However, in $Q$ or $R$, 
$a^{2}=a$ implies $a=0$ or $a=1$; as $a \neq 0$, we have $a=1$; thus, $x=I$ and $x$ is a nontrivial idempotent in $S$. Now, let $y=a+b I ; a \neq 0$ and $b \neq 0$ for $a=0$ will reduce to case $y=I$ is an idempotent.

$$
y^{2}=(a+b I) \times(a+b I)=a^{2}+b^{2} I+2 a b I
$$

That is, $y^{2}=a+b I \times a-b I=a^{2}+a b I+a b I+b^{2} I=a+b I$, equating the real and neutrosophic parts.

$$
a^{2}=a \text { i.e., } a(a-1)=0 \Rightarrow a=1 \text { as } a \neq 0 \text { and } 2 a b+b^{2}-b=0
$$

$b(2 a+b-1)=0 ; b \neq 0$, thus $2 a+b-1=0$; further, $a \neq 0$ as $a=0$ will reduce to the case $I^{2}=I$, thus $a=1$. Hence, $2+b-1=0$, thus $b=-1$. Hence, $a=1$ and $b=-1$ leading to $y=1-I$. Thus, only the non-trivial idempotents of $S$ are $I$ and $1-I$.

We next find the form of the triplets in S.

Theorem 2. Let $S=\{\langle Q \cup I\rangle,+, \times\}$ (or $\langle R \cup I\rangle,+, \times)$ be the neutrosophic ring. The neutrosophic triplets in $S$ are only of the following form for $a, b \in Q$ or $R$.

(i)

$$
\left(a-a I, 1-I, \frac{1}{a}-\frac{I}{a}\right) \text { and }\left(\frac{1}{a}-\frac{I}{a}, 1-I, a-a I\right) ; a \neq 0 .
$$

(ii)

$$
\left(b I, I, \frac{I}{b}\right) \text { and }\left(\frac{I}{b}, I, b\right) ; b \neq 0
$$

(iii)

$$
\left(a+b I, 1, \frac{1}{a}-\frac{b I}{a(a+b)}\right) ; a+b \neq 0 \text { and }\left(\frac{1}{a}-\frac{b I}{a(a+b)}, 1, a+b I\right) \text {. }
$$

Proof. Let $S$ be the neutrosophic ring. Let $x=\{a+b I, e+f I, c+d I\}$ be a neutrosophic triplet in $S ; a, b, c, d, e, f \in Q$ or $R$. We prove the neutrosophic triplets of $S$ are in one of the forms. If $x$ is a neutrosophic triplet, then we have

$$
\begin{aligned}
& a+b I \times e+f I=a+b I \\
& e+f I \times c+d I=c+d I
\end{aligned}
$$

and

$$
a+b I \times c+d I=e+f I
$$

Now, solving Equation (1), we get

$$
a e+(b f I+b e I+a f I)=a+b I
$$

Equating the real and neutrosophic parts, we get

$$
\begin{gathered}
a e=a \\
b f+b e+a f=b
\end{gathered}
$$

Expanding Equation (2), we get

$$
c e+f c I+d e I+f d I=c+d I .
$$

Equating the real and neutrosophic parts, we get

$$
c e=c
$$




$$
f c+d e+f d=d .
$$

Solving Equation (3), we get

$$
a c+b c I+b d I+a d I=e+f I
$$

Equating the real and neutrosophic parts, we get

$$
\begin{gathered}
a c=e \\
b c+b d+a d=f
\end{gathered}
$$

We find conditions so that Equations (4) and (5) are true.

Now, $a e=a$ and $b f+b e+a f=b$; $a e=a$ gives $a(e-1)=0$ if $a=0$ and $e \neq 1$ using in Equation (4), thus if $a=0$, we get $e=0$ and using $e=0$ in Equation (6), we get $c=0$. Thus, $a=c=e=0$. This forces $b \neq 0, d \neq 0$ and $f \neq 0$. We solve for $b, d$ and $f$ using Equations (5), (7) and (9). Equations (5) and (7) gives $b f=b$ as $b \neq 0, f=1$. Now, $f d=d$ as $f=1 ; d=d$. Equation (9) gives $b d=f$ or $b d=1$, thus

$$
d=\frac{1}{b}(b \neq 0) .
$$

Thus, we get

$$
\left(b I, I, \frac{I}{b}\right)
$$

to be neutrosophic triplet then

$$
\left(\frac{I}{b}, I, b I\right)
$$

is also a neutrosophic triplet. Thus, we have proved (ii) of the theorem.

Assume in Equation (4) $a e=a ; a \neq 0$, which forces $e=1$. Now, using Equation (8), we get $a c=1$, thus

$$
c=\frac{1}{a}
$$

Using Equation (5), we get $b f+b+a f=b$, thus $(a+b) f=0$. If $f=0$, then we have

$$
\left(a+b I, 1, \frac{1}{a}+d I\right)
$$

should be a neutrosophic triplet. That is,

$$
\begin{gathered}
(a+b I) \times\left(\frac{1}{a}+d I\right)=1 \\
1+\frac{b}{a} I+d a I+d b I=1 \\
\frac{b}{a}+d a+d b=0 \\
b+a^{2} d+a b d=0 \\
b(a d+1)+a^{2} d=0 \\
d\left(a^{2}+a b\right)=-b .
\end{gathered}
$$




$$
d=\frac{-b}{a^{2}+a b}=\frac{-b}{a(a+b)}
$$

$a \neq 0$ and $a+b \neq 0 . a+b \neq 0$ for if $a+b=0$, then $b=0$ we get $d=0$. Thus, the trivial triplet

$$
\left(a, 1, \frac{1}{a}\right)
$$

will be obtained. Thus, $a+b \neq 0$ and

$$
\left(a+b I, 1, \frac{1}{a}-\frac{b I}{a(a+b)}\right) \text { and }\left(\frac{1}{a}-\frac{b I}{a(a+b)}, 1, a+b I\right)
$$

are neutrosophic triplets so that Condition (iii) of theorem is proved.

Now, let $f \neq 0$, thus $a+b=0$ and $c+d=0$. We get $a=-b$ or $b=-a$ and $d=-c$. We have already proved $c=\frac{1}{a}$. Using Equations (8) and (9) and conditions $a=-b$ and $c=-d$, we get $f=-1$.

Hence, the neutrosophic triplets are

$$
\left(a-a I, 1-I, \frac{1}{a}-\frac{I}{a}\right) \text { and }\left(\frac{1}{a}-\frac{I}{a}, 1-I, a-a I\right)
$$

which is Condition (i) of the theorem.

Theorem 3. Let $S=\{\langle Q \cup I\rangle,+, \times\}($ or $\langle R \cup I\rangle,+, \times\})$ be the neutrosophic ring.

$$
M=\left\{\left(a-a I, 1-I, \frac{1}{a}-\frac{I}{a}\right) \mid a \in Q \backslash\{0\}\right\}
$$

be the collection of neutrosophic triplets of $S$ with neutral 1 - I is commutative group of infinite order with $(1-I, 1-I, 1-I)$ as the multiplicative identity.

Proof. To prove $M$ is a group of infinite order, we have to prove $M$ is closed under component-wise product and has an identity with respect to which every element has an inverse.

Let

$$
\begin{gathered}
x=\left(a-a I, 1-I, \frac{1}{a}-\frac{I}{a}\right) \text { and } y=\left(c-c I, 1-I, \frac{1}{c}-\frac{I}{c}\right) \in M \\
x \times y=\left(a-a I, 1-I, \frac{1}{a}-\frac{I}{a}\right) \times\left(c-c I, 1-I, \frac{1}{c}-\frac{I}{c}\right) \\
=\left(a c-a c I-a c I+a c I, 1-2 I+I, \frac{1}{a c}-\frac{I}{a c}-\frac{I}{a c}+\frac{I}{a c}\right) \\
=\left(a c-a c I, 1-I, \frac{1}{a c}-\frac{I}{a c}\right) \in M .
\end{gathered}
$$

Thus, $M$ is closed under component wise product.

We see that, when $a=1$, we get $e=(1-I, 1-I, 1-I) \in M$ is the identity of $M$ under component wise multiplication. Clearly, $e \times x=x \times e=x$ for all $x \in M$, thus $e$ is the identity of $M$. For every

$$
x=\left(a-a I, 1-I, \frac{1}{a}-\frac{I}{a}\right),
$$

we have a unique

$$
x^{-1}=\left(\frac{1}{a}-\frac{I}{a}, 1-I, a-a I\right) \in M
$$


such that

$$
\begin{gathered}
x \times x^{-1}=x^{-1} \times x=e=(1-I, 1-I, 1-I) \\
x \times x^{-1}=\left(a-a I, 1-I, \frac{1}{a}-\frac{I}{a}\right) \times\left(\frac{1}{a}-\frac{I}{a}\right)-\left(\frac{1}{a}-\frac{I}{a}, 1-I, a-a I\right) \\
=\left(\frac{a}{a}-\frac{a I}{a}-\frac{a I}{a}+\frac{a I}{a}, 1-2 I+I, \frac{a}{a}-\frac{a I}{a}-\frac{a I}{a}+\frac{a I}{a}\right) \\
=(1-I, 1-I, 1-I)
\end{gathered}
$$

as $a \neq 0$. Thus, $(M, \times)$ is a group under component wise product, which is known as the neutrosophic triplet group.

Theorem 4. Let $S=\{\langle Q \cup I\rangle,+, \times\}$ (or $\{\langle R \cup I\rangle,+, \times\})$ be the neutrosophic ring. The collection of neutrosophic triplets

$$
N=\left\{\left(a I, I, \frac{I}{a}\right) \mid a \in Q \backslash\{0\}\right\}
$$

(or $R \backslash\{0\}$ ) forms a commutative group of infinite order under component wise multiplication with (I, I, I) as the multiplicative identity.

Proof. Let

$$
N=\left\{\left(a I, I, \frac{I}{a}\right) \mid a \neq 0 \in Q \text { or } R\right\}
$$

be a collection of neutrosophic triplets. To prove $N$ is commutative group under component wise product, let

$$
x=\left(a I, I, \frac{I}{a}\right)
$$

and

$$
y=\left(b I, I, \frac{I}{b}\right) \in M
$$

To show $x \times y \in N$.

$$
x \times y=\left(a I, I, \frac{I}{a}\right) \times\left(b I, I, \frac{I}{b}\right)=\left(a b I, I, \frac{I}{a b}\right),
$$

using the fact $I^{2}=I$. Hence, $(N, \times)$ is a semigroup under product.

Considering $e=(I, I, I) \in N$, we see that $e \times e=x \times e=x$ for all $x \in N$.

$$
e \times x=(I, I, I) \times\left(a I, I, \frac{I}{a}\right)=\left(a I, I, \frac{I}{a}\right)=x\left(\text { using } I^{2}=I\right) .
$$

Thus, $(I, I, I)$ is the identity element of $(N, \times)$. For every

$$
x=\left(a I, I, \frac{I}{a}\right),
$$

we have a unique

$$
x^{-1}=\left(\frac{I}{a}, I, a\right) \in N
$$

is such that

$$
x \times x^{-1}=\left(a I, I, \frac{I}{a}\right)=(I, I, I)
$$

as $a \neq 0$ and $I^{2}=I$.

Thus, $\{N, \times\}$ is a commutative group of infinite order. 
It is interesting to note both the sets $\mathrm{M}$ and $\mathrm{N}$ are not even closed under addition.

Next, let

$$
P=\left\{a+b I, 1, \frac{1}{a}-\frac{b I}{a(a+b)} ; a \neq b ; a+b \neq 0, a \neq 0 .\right\}
$$

We get

$$
a+b I \times \frac{1}{a}-\frac{b I}{a(a+b)}=1 .
$$

We call these neutrosophic triplets as special neutrosophic triplets contributed by the unity 1 of the ring which is the trivial idempotent of $S$; however, where it is mandatory, $x$ and anti $(x)$ are nontrivial neutrosophic numbers with neut $(x)=1$.

Theorem 5. Let $S=\langle Q \cup I\rangle,+, \times($ or $\langle R \cup I\rangle,+, \times)$ be the neutrosophic ring. Let

$$
P=\left\{\left(a+b I, 1, \frac{1}{a}-\frac{b I}{a(a+b)} ; a \neq b, \text { where } a, b \in Q \backslash\{0\}(\text { or } R \backslash 0) \text { and } a+b \neq 0\right\}\right.
$$

be the collection of special neutrosophic triplets with 1 as the neutral. $P$ is a torsion free abelian group of infinite order with $(1,1,1)$ as its identity under component wise product.

Proof. It is easily verified $P$ is closed under the component wise product and $(1,1,1)$ acts as the identity for component wise product. For every

$$
x=\left(a-b I, 1, \frac{1}{a}+\frac{b I}{a(a-b)}\right) \in P,
$$

we have a unique

$$
y=\left(\frac{1}{a}+\frac{b I}{a(a-b)}, 1, a-b I\right) \in P
$$

such that $x \times y=(1,1,1)$. We also see $x^{n} \neq(1,1,1)$ for any $x \in P$ and $n \neq 0(n>0) ; x \neq(1,1,1)$, hence $P$ is a torsion free abelian group.

\section{Discussion and Conclusions}

We show that, in the case of neutrosophic duplets in $\langle Z \cup I\rangle,\langle Q \cup I\rangle$ or $\langle R \cup I\rangle$, the collection of duplets $\{a-a I\}$ forms a neutrosophic subring. However, in the case of neutrosophic triplets, we show that $\langle Z \cup I\rangle$ has no nontrivial triplets and we have shown there are three distinct collection of neutrosophic triplets in $\langle R \cup I\rangle$ and $\langle Q \cup I\rangle$. We have proved there are only three types of neutrosophic triplets in these neutrosophic rings and all three of them form abelian groups that are torsion free under component wise product. For future research, we would apply these neutrosophic triplets to concepts akin to SVNS and obtain some mathematical models.

Author Contributions: Conceptualization, V.K.W.B. and F.S.; writing-original draft preparation, V.K.W.B. and I.K.; writing-review and editing, I.K.

Funding: This research received no external funding.

Acknowledgments: The authors would like to thank the reviewers for their reading of the manuscript and many insightful comments and suggestions.

Conflicts of Interest: The authors declare no conflict of interest.

\section{References}

1. Smarandache, F. A Unifying Field in Logics: Neutrosophic Logic. Neutrosophy, Neutrosophic Set, Neutrosophic Probability and Statistics; American Research Press: Rehoboth, DE, USA, 2005; ISBN 978-1-59973-080-6. 
2. Smarandache, F. Neutrosophic set-a generalization of the intuitionistic fuzzy set. In Proceedings of the 2006 IEEE International Conference on Granular Computing, Atlanta, GA, USA, 10-12 May 2006; pp. $38-42$.

3. Wang, H.; Smarandache, F.; Zhang, Y.; Sunderraman, R. Single valued neutrosophic sets. Review 2010, 1, $10-15$.

4. Kandasamy, I. Double-Valued Neutrosophic Sets, their Minimum Spanning Trees, and Clustering Algorithm. J. Intell. Syst. 2018, 27, 163-182. [CrossRef]

5. Kandasamy, I.; Smarandache, F. Triple Refined Indeterminate Neutrosophic Sets for personality classification. In Proceedings of the 2016 IEEE Symposium Series on Computational Intelligence (SSCI), Athens, Greece, 6-9 December 2016; pp. 1-8. [CrossRef]

6. Vasantha, W.B.; Smarandache, F. Basic Neutrosophic Algebraic Structures and Their Application to Fuzzy and Neutrosophic Models; Hexis: Phoenix, AZ, USA, 2004; ISBN 978-1-931233-87-X.

7. Vasantha, W.B.; Smarandache, F. N-Algebraic Structures and SN-Algebraic Structures; Hexis: Phoenix, AZ, USA, 2005; ISBN 978-1-931233-05-5.

8. Vasantha, W.B.; Smarandache, F. Some Neutrosophic Algebraic Structures and Neutrosophic N-Algebraic Structures; Hexis: Phoenix, AZ, USA, 2006; ISBN 978-1-931233-15-2.

9. Vasantha, W.B.; Smarandache, F. Neutrosophic Rings; Hexis: Phoenix, AZ, USA, 2006; ISBN 978-1-931233-20-9.

10. Agboola, A.A.A.; Adeleke, E.O.; Akinleye, S.A. Neutrosophic rings II. Int. J. Math. Comb. 2012, 2, 1-12.

11. Smarandache, F. Operators on Single-Valued Neutrosophic Oversets, Neutrosophic Undersets, and Neutrosophic Offsets. J. Math. Inf. 2016, 5, 63-67. [CrossRef]

12. Smarandache, F.; Ali, M. Neutrosophic triplet group. Neural Comput. Appl. 2018, 29, 595-601. [CrossRef]

13. Agboola, A.A.A.; Akinola, A.D.; Oyebola, O.Y. Neutrosophic Rings I. Int. J. Math. Comb. 2011, 4, 115.

14. Ali, M.; Smarandache, F.; Shabir, M.; Naz, M. Soft Neutrosophic Ring and Soft Neutrosophic Field. Neutrosophic Sets Syst. 2014, 3, 53-59.

15. Ali, M.; Smarandache, F.; Shabir, M.; Vladareanu, L. Generalization of Neutrosophic Rings and Neutrosophic Fields. Neutrosophic Sets Syst. 2014, 5, 9-13.

16. Ali, M.; Shabir, M.; Smarandache, F.; Vladareanu, L. Neutrosophic LA-semigroup Rings. Neutrosophic Sets Syst. 2015, 7, 81-88.

17. Broumi, S.; Smarandache, F.; Maji, P.K. Intuitionistic Neutrosphic Soft Set over Rings. Math. Stat. 2014, 2, 120-126.

18. Smarandache, F. Neutrosophic Perspectives: Triplets, Duplets, Multisets, Hybrid Operators, Modal Logic, Hedge Algebras and Applications, 2nd ed.; Pons Publishing House: Brussels, Belgium, 2017; ISBN 978-1-59973-531-3.

19. Sahin, M.; Abdullah, K. Neutrosophic triplet normed space. Open Phys. 2017, 15, 697-704. [CrossRef]

20. Smarandache, F. Hybrid Neutrosophic Triplet Ring in Physical Structures. Bull. Am. Phys. Soc. 2017, $62,17$.

21. Smarandache, F.; Ali, M. Neutrosophic Triplet Field used in Physical Applications. In Proceedings of the 18th Annual Meeting of the APS Northwest Section, Pacific University, Forest Grove, OR, USA, 1-3 June 2017.

22. Smarandache, F.; Ali, M. Neutrosophic Triplet Ring and its Applications. In Proceedings of the 18th Annual Meeting of the APS Northwest Section, Pacific University, Forest Grove, OR, USA, 1-3 June 2017.

23. Zhang, X.H.; Smarandache, F.; Liang, X.L. Neutrosophic Duplet Semi-Group and Cancellable Neutrosophic Triplet Groups. Symmetry 2017, 9, 275. [CrossRef]

24. Bal, M.; Shalla, M.M.; Olgun, N. Neutrosophic Triplet Cosets and Quotient Groups. Symmetry 2017, 10, 126. [CrossRef]

25. Zhang, X.H.; Smarandache, F.; Ali, M.; Liang, X.L. Commutative neutrosophic triplet group and neutro-homomorphism basic theorem. Ital. J. Pure Appl. Math. 2017. [CrossRef]

26. Vasantha, W.B.; Kandasamy, I.; Smarandache, F. Neutrosophic Triplet Groups and Their Applications to Mathematical Modelling; EuropaNova: Brussels, Belgium, 2017; ISBN 978-1-59973-533-7.

27. Vasantha, W.B.; Kandasamy, I.; Smarandache, F. A Classical Group of Neutrosophic Triplet Groups Using $\left\{Z_{2 p}, \times\right\}$. Symmetry 2018, 10, 194. [CrossRef]

28. Vasantha, W.B.; Kandasamy, I.; Smarandache, F. Neutrosophic duplets of $\left\{Z_{p n}, \times\right\}$ and $\left\{Z_{p q}, \times\right\}$. Symmetry 2018, 10, 345. [CrossRef]

29. Vasantha, W.B.; Kandasamy, I.; Smarandache, F. Algebraic Structure of Neutrosophic Duplets in Neutrosophic Rings $\langle Z \cup I\rangle,\langle Q \cup I\rangle$ and $\langle R \cup I\rangle$. Neutrosophic Sets Syst. 2018, 23, 85-95.

30. Vasantha, W.B.; Kandasamy, I.; Smarandache, F. Semi-Idempotents in Neutrosophic Rings. Mathematics 2019, 7, 507. [CrossRef] 
31. Smarandache, F.; Zhang, X.; Ali, M. Algebraic Structures of Neutrosophic Triplets, Neutrosophic Duplets, or Neutrosophic Multisets. Symmetry 2019, 11, 171. [CrossRef]

32. Zhang, X.H.; Wu, X.Y.; Smarandache, F.; Hu, M.H. Left (right)-quasi neutrosophic triplet loops (groups) and generalized BE-algebras. Symmetry 2018, 10, 241. [CrossRef]

33. Zhang, X.H.; Wang, X.J.; Smarandache, F.; Jaíyéolá, T.G.; Liang, X.L. Singular neutrosophic extended triplet groups and generalized groups. Cognit. Syst. Res. 2018, 57, 32-40. [CrossRef]

34. Zhang, X.H.; Wu, X.Y.; Mao, X.Y.; Smarandache, F.; Park, C. On Neutrosophic Extended Triplet Groups (Loops) and Abel-Grassmann's Groupoids (AG-Groupoids). J. Intell. Fuzzy Syst. 2019. [CrossRef]

35. Zhang, X.; Hu, Q.; Smarandache, F.; An, X. On Neutrosophic Triplet Groups: Basic Properties, NT-Subgroups, and Some Notes. Symmetry 2018, 10, 289. [CrossRef]

36. Ma, Y.; Zhang, X.; Yang, X.; Zhou, X. Generalized Neutrosophic Extended Triplet Group. Symmetry 2019, 11, 327. [CrossRef]

37. Kanel-Belov, A.; Halle Rowen, L. Computational Aspects of Polynomial Identities; Research Notes in Mathematics; CRC Press: Boca Raton, FL, USA, 2005; ISBN 9781568811635.

(C) 2019 by the authors. Licensee MDPI, Basel, Switzerland. This article is an open access article distributed under the terms and conditions of the Creative Commons Attribution (CC BY) license (http://creativecommons.org/licenses/by/4.0/). 